\title{
Comparative performance of prediction model, non-expert and tele-diagnosis of common external and middle ear disorders using a cohort of 85 patients from Cambodia
}

\author{
James Schuster-Bruce ${ }^{1,2}$, Prajwal Shetty ${ }^{3}$, James O’Donovan ${ }^{4}$, Rishi Mandavia ${ }^{5}$, Touch \\ Sokdavy $^{6}$, and Mahmood Bhutta ${ }^{7}$ \\ ${ }^{1}$ St George's, University of London \\ ${ }^{2}$ St George's University Hospital NHS Foundation Trust \\ ${ }^{3}$ Worthing Hospital \\ ${ }^{4}$ University of Oxford \\ ${ }^{5}$ National Institute for Health Research \\ ${ }^{6}$ The Children's Surgical Centre \\ ${ }^{7}$ Brighton and Sussex University Hospitals NHS Trust
}

June 17,2020

\begin{abstract}
Key Points: 1. Efforts to combat the large global burden of ear and hearing disorders are hampered by poor availability of expert diagnosis 2 . We report the first study to directly compare prediction model, non-expert and tele-diagnosis of middle and external ear disorders. 3. A prediction model based upon a novel automated otological symptom questionnaire performed poorly, but absence of otorrhoea was found to reliably exclude a diagnosis of chronic suppurative otitis media. 4. Both on-site non-expert and expert tele-diagnosis had high diagnostic specificity, but low sensitivity. 5. Future work could explore how the validity of these diagnostic methods may be improved.
\end{abstract}

\section{Key Points:}

1. Efforts to combat the large global burden of ear and hearing disorders are hampered by poor availability of expert diagnosis

2. We report the first study to directly compare prediction model, non-expert and tele-diagnosis of middle and external ear disorders.

3. A prediction model based upon a novel automated otological symptom questionnaire performed poorly, but absence of otorrhoea was found to reliably exclude a diagnosis of chronic suppurative otitis media.

4. Both on-site non-expert and expert tele-diagnosis had high diagnostic specificity, but low sensitivity.

5. Future work could explore how the validity of these diagnostic methods may be improved.

\section{Introduction}

Globally $6 \%$ of the population, or around 466 million people, suffer from disabling hearing loss (www.who.int/pbd/deafness/estimates/en/), and the majority reside in low- and middle-income countries. One barrier to tackling this large burden is the lack of specialist health workers to recognise and manage ear and hearing disorders. ${ }^{1}$

Fuelled by technological advances, there is growing interest in remote or non-expert diagnosis in ear and hearing care. However, studies to date have been limited, mostly focused on screening for hearing loss, 
without exploring additional symptoms or signs which may signify middle or external ear disorders. This is important: of those suffering disabling hearing loss, around half (200 million) are thought to be due to chronic suppurative otitis media (CSOM) ${ }^{2}$ where surgical intervention may be indicated.

Diagnosis of external and middle ear disorders is based on clinical history, including symptoms of otalgia, otorrhoea, and hearing loss, supplemented by otoscopy to visualise the ear canal and tympanic membrane. Diagnostic accuracy relates to expertise and experience of the assessor. Specialist assessment with appropriate tools (including a good quality otoscope) is the gold standard, but due to resource gaps in equipment and specialist health personnel, is not universally available.

We recognise three alternative strategies (Figure 1):

- Telediagnosis ${ }^{3}$ where relevant diagnostic information is captured (usually by a non-specialist) and sent to a specialist

- Non-expert diagnosis, where a non-specialist (defined as someone with less training or experience than a specialist) makes a diagnosis.

- Prediction model diagnosis, ${ }^{4}$ where data from a diagnostic tool is fed into an algorithm, with no or minimal human interpretation

Figure 1: Diagram showing gold standard assessment and three further strategies for diagnosis: tele-diagnosis, non-specialist diagnosis and prediction model diagnosis.

To date few studies have explored the validity or feasibility of such approaches for diagnosis of external and middle ear disease.

Tele-diagnosis using otoscopic images captured with an endoscope or smartphone has been shown to be successful if users are trained in image capture, ${ }^{5}$ but appears unsuccessful if users are given only instructions on use. ${ }^{6,7}$ The requirement for training may therefore limit the scalability of this approach.

Studies of non-expert diagnosis of outer and middle ear disorders are sparse, but results to date are not encouraging. In high income settings, medical students, audiology students and general practitioners report low self-confidence in diagnostic otoscopy. ${ }^{8} \mathrm{~A}$ recent study found poor reliability of nurse otoscopic diagnosis in Malawi. ${ }^{9}$ Validity of non-expert diagnosis therefore remains uncertain.

Prediction model diagnosis is largely unexplored in this field. For example (and to our knowledge) no study has explored if an algorithm using patient history is valid to diagnose or differentiate external or middle ear disorders.

Here we devised a novel study to directly compare the utility of tele-diagnosis, non-expert diagnosis, and prediction model diagnosis as a screening tool for common external and middle ear disorders. Our test bed was a cohort of patients presenting to an Ear, Nose and Throat (ENT) clinic in [removed for blind peer review] hospital in Cambodia (www.csc.org), a setting with high prevalence of otological disease.

\section{Methods}

Ethics

The ethics board of [removed for blind peer review] granted approval.

\section{Participants}

We invited participation from consenting consecutive adult and paediatric patients presenting with selfdeclared ear or hearing symptoms to ENT outpatients at [removed for blind peer review] hospital between 21st - 30th May 2018.

\section{Data capture}

Each participant underwent sequential assessment using platforms to capture data on symptoms and otoscopic appearance (figure 2). 
Figure 2: Diagrammatic representation of study design.

First, symptom history was captured using an automated questionnaire (devised by author [removed for blind peer review] in English, piloted in ENT clinics in Tanzania and England, and then translated into Khmer). An initial question asked participants to report ear discharge (otorrhoea), ear pain (otalgia) and/or hearing loss (we also captured symptoms of dizziness and tinnitus, but did not use these in subsequent analysis). Forced responses and skip logic (figure 3) were used to further define each symptom, including laterality, chronology, and (for otorrhoea) consistency. The algorithm also collected date on demographical information, past medical and surgical history and drug and allergy history. For this study the questionnaire was hosted on the Cupris platform (Cupris Ltd, London), and data were captured by one of three ENT specialist nurses who were native Khmer language speakers and had worked at CSC hospital for 1-6 years.

Figure 3: Diagrams displaying symptom-specific algorithms for (left to right) ear pain, runny ear and hearing loss. Demographic data, as well as past medical history, drug history and allergies were also collected by the questionnaire.

Next, the same nurse administering the questionnaire captured brief otoscopic videos of each ear using the Cupris TYM device (a platform we have previously shown to be valid for expert tele-diagnosis ${ }^{5}$ ) attached to an Apple iPhone 5S mobile phone (Apple Inc., Cupertino). Videos were encrypted and stored. Because nurses were naïve to the device, they were given a short tutorial (author [removed for blind peer review]) before the study commenced, and trialled the device on ten consenting individuals.

Finally, each participant underwent assessment by one of two resident ENT specialists. They took a targeted clinical history (with open responses) in the Khmer language, performed oto-endoscopy, and recorded their diagnosis. In cases where wax precluded a full oto-endoscopic view, data were removed from final analyses. Specialist assessment was blind to previous assessment, and conducted in a separate area.

\section{Outputs}

Captured data were used to ascertain three outputs for non-expert diagnosis (figure 2).

The first output was data captured by the questionnaire, which was used for prediction model diagnosis. We also recorded time taken to administer the questionnaire.

The second output was diagnosis reached by the non-expert (nurse), using data they captured with the questionnaire and video otoscopy on the Cupris device. They were asked to select a diagnosis from a list: normal / acute otitis media (AOM) / CSOM / inactive tympanic membrane perforation / otitis externa / otitis media with effusion / cholesteatoma / other.

The third output was tele-diagnosis by an expert, using data captured by the non-expert (nurse). Questionnaire results were translated back into English, and these and the otoscopic videos were anonymised and sent to specialist otologists in the UK (authors [removed for blind peer review] and [removed for blind peer review]). Owing to logistical constraints, data were reviewed 9-11 months after capture. Experts were asked to select a diagnosis from the same list as the nurse.

All assessments were blind to diagnosis by other methods.

\section{Comparison to gold standard}

Performance of each diagnostic method was compared to the gold standard, which here was diagnosis by the resident ENT specialist. For each method we calculated sensitivity, specificity, positive and negative predictive value, and likelihood ratio. For the questionnaire we evaluated performance of: each major symptom in isolation; sub-characteristics of each symptom; and combinations of major symptoms. For analysis we classified each ear as independent.

\section{Results}


We recruited 85 participants ( 15 children and 70 adults), giving a total of 170 ears. One case was lost-to-follow up.

Completion of the questionnaire took an average of 6 minutes (range 1-14 minutes). In 23 ears wax precluded a diagnosis, and in a further 9 , technical faults interrupted data capture. Therefore, we analysed questionnaire data for 138 ears.

For otoscopy, we obtained video data for 138 ears. However, experts considered three videos inadequate for tele-diagnosis (poor view of ear canal and/or tympanic membrane), leaving 135 cases for this method.

Gold standard diagnosis was achieved in all remaining cases, with 56 classified as normal external and middle ear, 22 CSOM, 21 otitis externa, 9 inactive tympanic membrane perforation, and 4 AOM. Performance of each diagnostic method compared to the gold standard is summarised in Table 1.

For prediction model diagnosis using the questionnaire, no particular symptom or combination of symptoms reliably differentiated diagnostic categories. For a symptom in isolation, sensitivity for a named diagnosis varied from 33-76\%, except for presence of otorrhoea, which had $95 \%$ sensitivity for CSOM. Specificity varied between $67-80 \%$.

Non-expert (nurse) diagnosis performed well for specificity (90-99\% for all diagnoses) but poorly for sensitivity ( $<43 \%$ for all diagnoses).

Remote expert diagnosis also performed well for specificity (86-99\%), but again less well for sensitivity (32-100\%).

\section{Discussion}

This is the first study to directly compare performance of different methods for diagnosis of external and middle ear disorders. We report variable results. Prediction model diagnosis using automated history performed poorly, but an absence of otorrhoea (perhaps unsurprisingly) was found to reliably exclude CSOM. This implies that individuals who deny a history of ear discharge could confidently be excluded in programs screening for CSOM.

Whereas both nurse-led and remote expert diagnosis showed high specificity, they had low sensitivity. The reasons for poor sensitivity can only be surmised, but could include poor performance of the questionnaire, poor quality video capture on otoscopy, insufficient video resolution, low experience or confidence of the assessor, or diagnostic uncertainty or disagreement. Poor image capture and diagnostic incongruity has been reported in other studies. ${ }^{7}$

We recognise our study as a preliminary exploration of methods for screening or diagnosis of middle or external ear disease (where on-site specialist assessment is unavailable). Future work could look to evaluate if and how validity of these approaches may be improved, including through improving the quality or platform for image capture to assist remote diagnosis; targeted training of non-experts to develop diagnostic skills; or providing additional or enhanced information to facilitate prediction model diagnosis (in particular the addition of automated interpretation of otoscopic images). ${ }^{10}$

\section{Conclusions}

We report the first study to directly compare three approaches for non-specialist diagnosis of disorders of the middle or external ear. Our results show suboptimal but comparable performance using an automated questionnaire, on site non-expert diagnosis, or remote expert diagnosis. Future work should look to enhance the performance of these approaches, including through training of non-experts, or through enhanced algorithms for diagnosis including incorporation of automated otoscopy.

\section{References:}

1. Bhutta MF, Bu X, de Muñoz PC, Garg S, Kong K. Training for hearing care providers. Bull World Health Organ. 2019 Oct 1;97(10):691-8. 
2. World Health Organization. Chronic suppurative otitis media : burden of illness and management options [Internet]. 2004 [cited 2020 May 31]. Available from: https://www.who.int/pbd/publications/Chronicsuppurativeotitis_media.pdf?ua=1

3. Hersh WR, Hickam DH, Severance SM, Dana TL, Krages KP, Helfand M. Diagnosis, access and outcomes: Update of a systematic review of telemedicine services. J Telemed Telecare. 2006 Sep;12(2_suppl):3-31.

4. Collins GS, Reitsma JB, Altman DG, Moons KGM. Transparent reporting of a multivariable prediction model for individual prognosis or diagnosis (TRIPOD): the TRIPOD statement. BMJ. 2015 Jan 7;350(jan07 4):g7594-g7594.

5. Mandavia R, Lapa T, Smith M, Bhutta MF. A cross-sectional evaluation of the validity of a smartphone otoscopy device in screening for ear disease in Nepal. Clin Otolaryngol. 2016;43(1):31-8.

6. Erkkola-Anttinen N, Irjala H, Laine MK, Tahtinen PA, Loyttyniemi E, Ruohola A. Smartphone Otoscopy Performed by Parents. Telemedicine and e-Health. 2019 Jun;25(6):477-84.

7. Demant MN, Jensen RG, Bhutta MF, Laier GH, Lous J, Homoe P. Smartphone otoscopy by nonspecialist health workers in rural Greenland: A cross-sectional study. International Journal of Pediatric Otorhinolaryngology. 2019 Nov;126:109628.

8. Niermeyer WL, Philips RHW, Essig GF, Moberly AC. Diagnostic accuracy and confidence for otoscopy: Are medical students receiving sufficient training?: Otoscopic Accuracy and Confidence. The Laryngoscope. 2019 Aug;129(8):1891-7.

9. Bright T, Mulwafu W, Phiri M, Ensink RJH, Smith A, Yip J, et al. Diagnostic accuracy of non-specialist versus specialist health workers in diagnosing hearing loss and ear disease in Malawi. Trop Med Int Health. 2019 May 9;tmi.13238.

10. Livingstone D, Talai AS, Chau J, Forkert ND. Building an Otoscopic screening prototype tool using deep learning. J of Otolaryngol - Head \& Neck Surg. 2019 Dec;48(1):66.

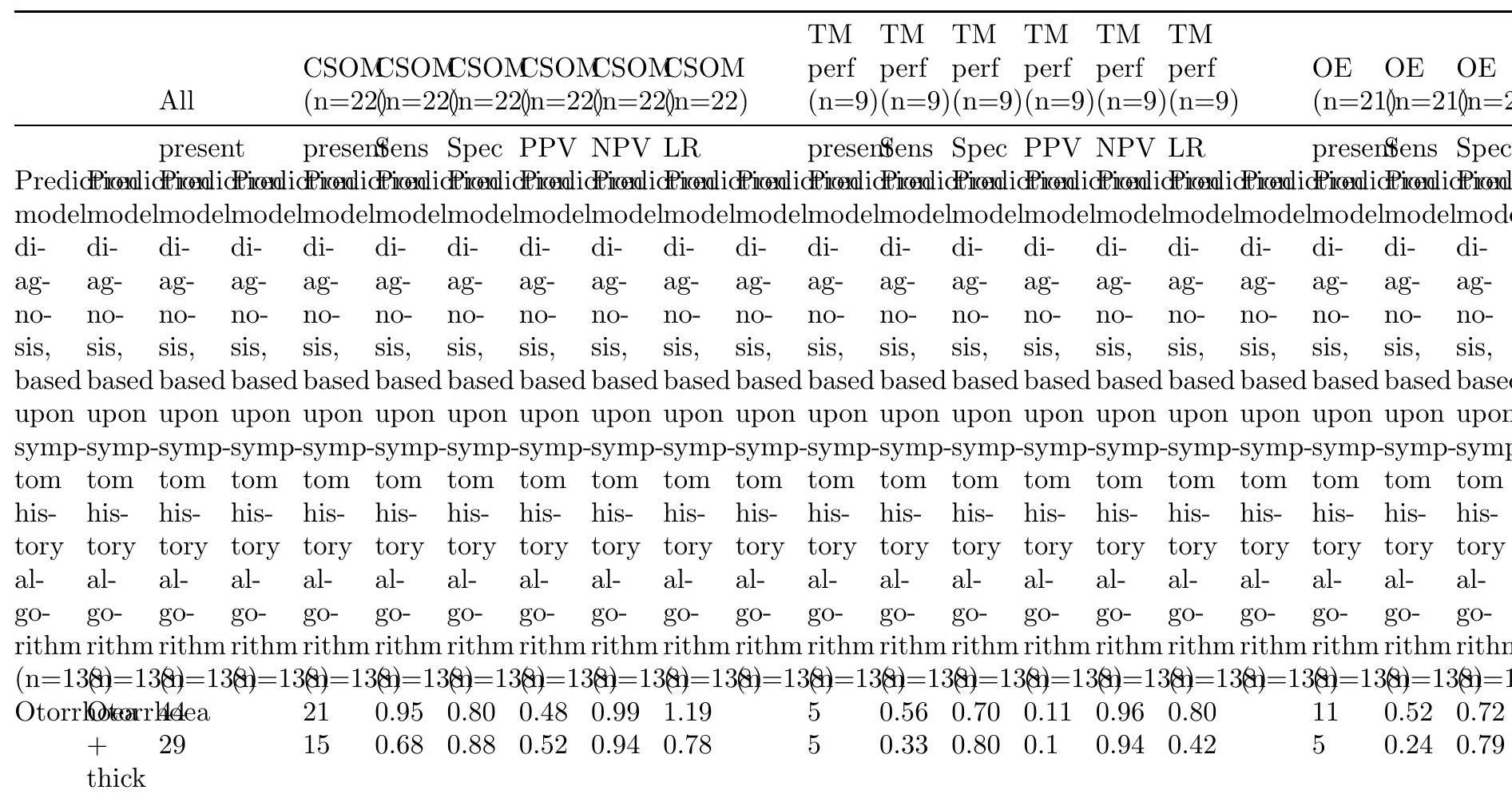




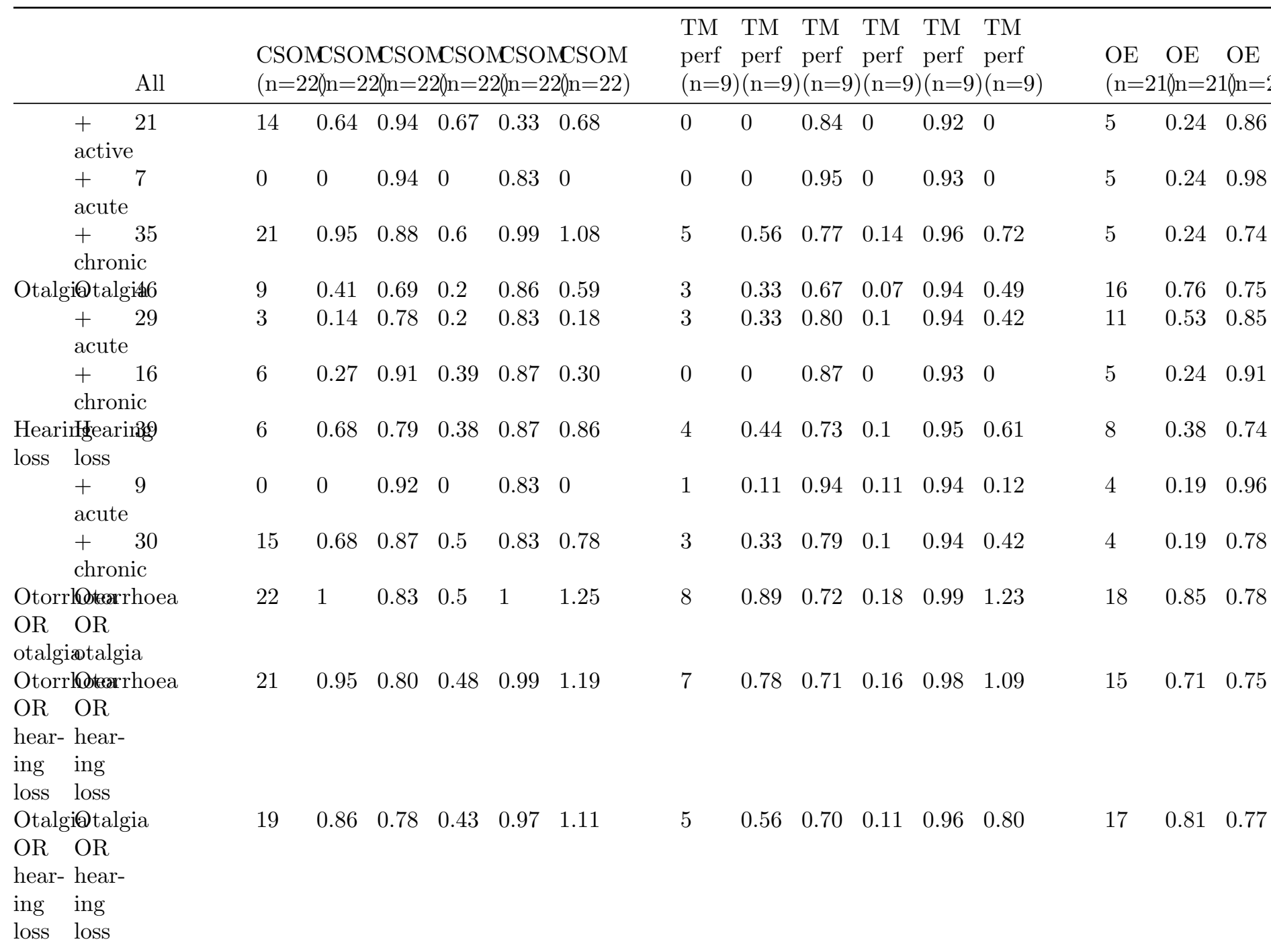




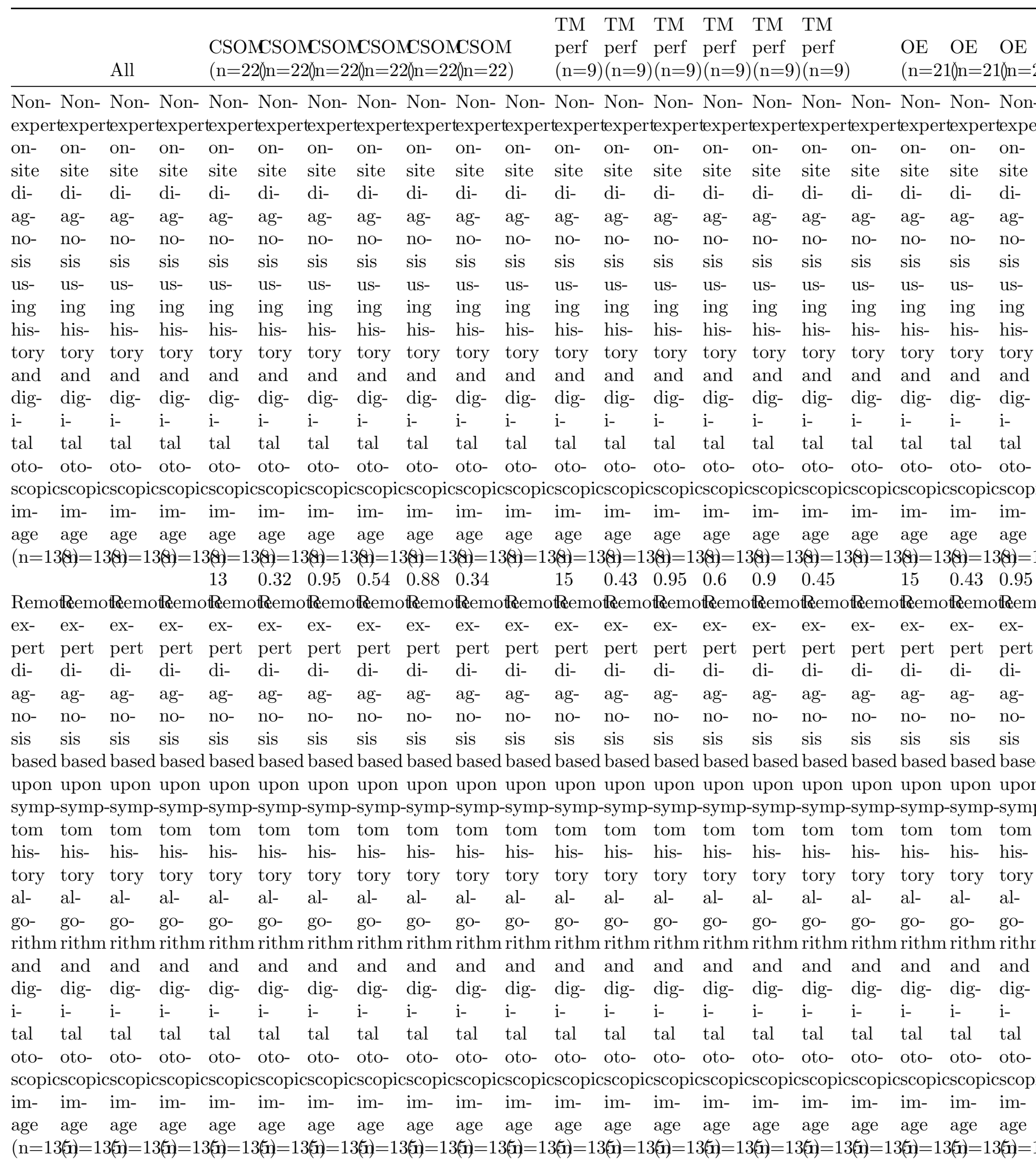




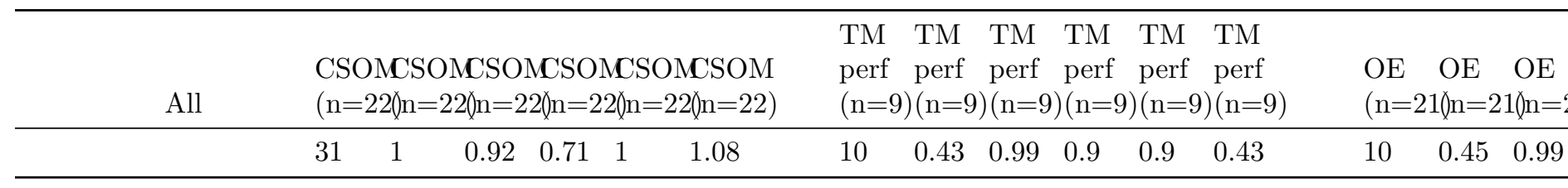

Table 1: Table displaying sensitivity (sens), specificity (spec), positive predictive values (PPV), negative predictive values (NPV) and likelihood ratios (LR) across diagnoses for three different diagnostic models. Diagnoses include Chronic Supparative Otitis Media (CSOM), Inactive TM perforation (TM perf), Otitis Externa (OE) and Acute Otitis Media (AOM).
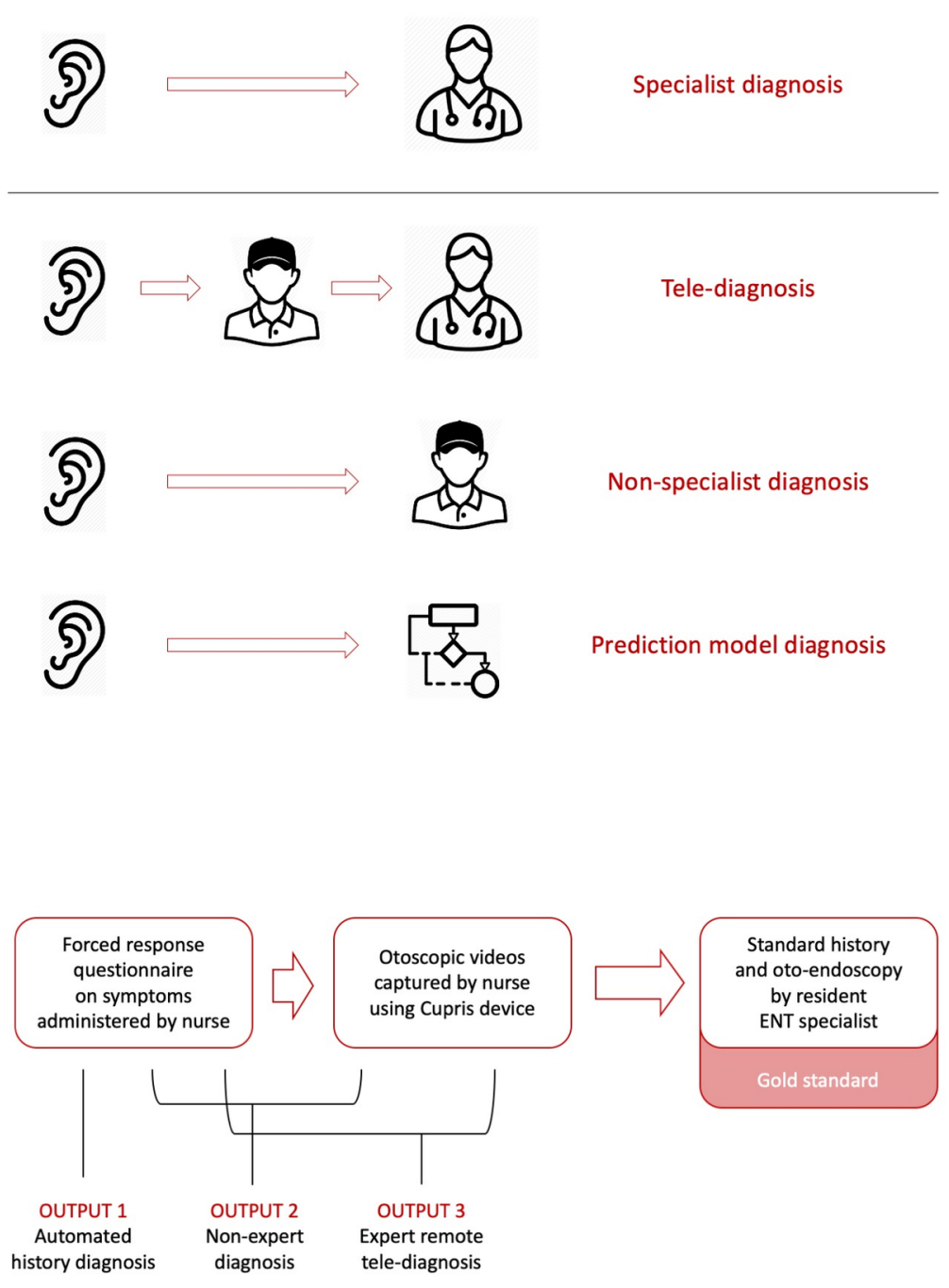
a)

1. Which ear is Runny?

a.Left

b.Right

c. Both (algorithm asks the following questions

$\downarrow$ specific to right ear and then left ear)

2. What is the type of discharge that comes out of the ear?

a. Thick, Yellow and sticky

b. Watery and white/clear

c. Thick and sometimes with blood

$\downarrow$ d. Other- please specify (free text)

3. Is your ear discharging now?

a. Yes

$\downarrow$ b. No (go to 6)

4. Thinking about this episode how long has your ear been runny (discharging) for?

a. only a few days

b. a few weeks

c. months or years

5. Have you had any treatment for this episode of runny (discharging) ear?

$$
\begin{aligned}
& \text { a. No } \\
& \text { b. Yes- antibiotic drops into the ear } \\
& \text { c. Yes- antibiotic drops by mouth } \\
& \text { d. Yes- antibiotic drops and by mouth } \\
& \text { e. other- please specify (free text) }
\end{aligned}
$$

6. Thinking about the first time you had runny (discharging) ear, when was that?

a. I did not have any problem with runny ears

until a few days ago

b. I first had a runny ear a few months ago

c. I first had a runny ear a few years ago

$\downarrow$ d. I first had a runny ear many years ago

7. How often do you get runny (discharging ear)?

a. This is the first time I have had a runny ear

b. I will usually get a runny ear daily

c. I will usually get a runny ear every few weeks

d. I will usually get a runny ear every few months

e. It is usually more than 6 months between episodes of runny ear

f. my ear is always runny

8. When you have and a runny (discharging) ear before what treatment do you normally get?

a. antibiotics from my doctor

b. antibiotics by mouth

c. No treatment- it goes away by itself

d. No treatment - and its still runny

e. Other- please specify (free text)

9. When you get a runny ear is often after swimming or washing?

a. yes

$\downarrow$ b. no

10. Is there anything else you want to tell us about your runny

(discharging) ear? 
b)

1. Which ear has pain?

Right

Left

Both (Algorithm asks the following questions

specific to right ear and then left ear)

2. How long have you suffered with ear pain?

A few days (go to 4 )

A few weeks

Months or years

3. How often do you get the pain?

All the time

Several times a day

Every few days

Once every few weeks

Once every few months

Less often

4. How would you describe the pain?

A shooting pain that lasts (a few seconds)

A prolonged pain (minutes or hours)

$\downarrow$ Other (please specify)

5. When you have the pain, how would you describe it's intensity?

A mild discomfort that is bearable

A pain that troubles me a little

Very painful

Severe pain

6. Does the pain occur associated with any other symptoms?

No- I only have ear pain

Yes- I get ear pain when I also have ear discharge

Yes- when I get pain I have a fever

Yes- I get the pain when I open my mouth or chew

Yes- I get pain when I have a sore throat

Yes- other

7. Is there anything else you would like to tell us about your ear pain? 
c) 1. Which ear has poor hearing?

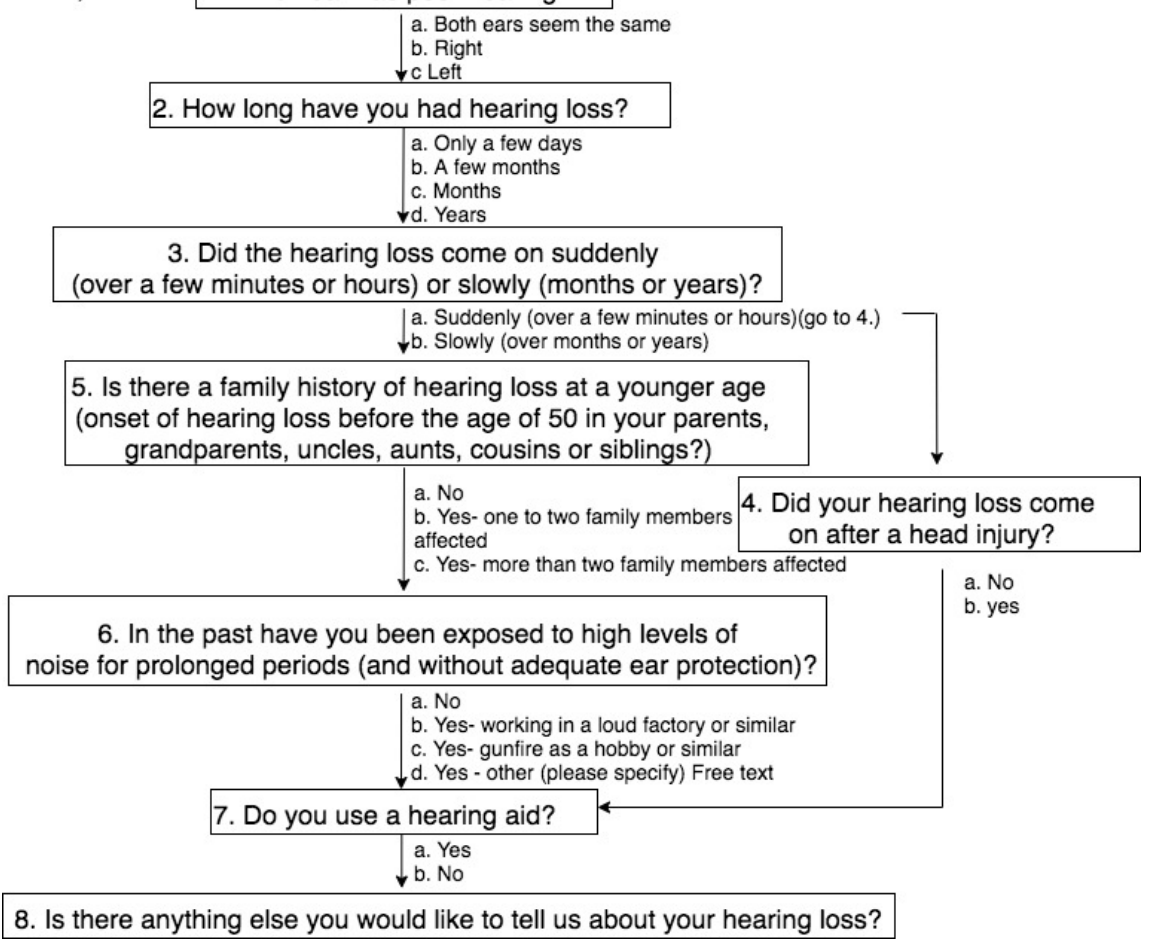

